\title{
Simulation of 2D Heat Transfer in a Horizontal Plane with Thermal Resistance-Capacitance Model
}

\author{
Eric Rohrbach ${ }^{1,2}$, Lanbo Liu ${ }^{1,3, *}$ \\ ${ }^{1}$ Center for Integrative Geosciences, University of Connecticut \\ 354 Mansfield Road, Storrs, CT 06269-1045, USA \\ Eric.Rohrbach@uconn.edu, Lanbo.Liu@uconn.edu* \\ ${ }^{2}$ United Technology Research Center \\ 411 Silver Ln, East Hartford, CT 06118, USA \\ ${ }^{3}$ Dept. of Civil \& Environmental Engineering, University of Connecticut \\ 261 Glenbrook Road, U-3037, Storrs, CT 06269-3037, USA
}

\begin{abstract}
In this paper, a thermal resistance capacitance model (TRCM) technique is developed for studying the heat transport in the horizontal plane of a borehole heat exchanger (BHE) array for a seasonal thermal energy storage (STES) system. We started from the TRCM model previously developed from other studies for a single BHE borehole and expanded it to have an azimuthal sense with a hexagonal element to represent a single BHE. Then we use it as an element to form a honey comb shaped collections to model the heat transport in a horizontal plane of a BHE array at a representative depth by solving an ordinary differential equation set. We validated the approach by comparison with the results generated by the finite element method for the same model and the results have reached excellent agreement. The major findings and conclusive remarks are: 1) a novel TRCM model is developed with the capability to model heat transport in a horizontal plane for the study of the temperature evolution in the soil formations in a STEMM BHE system; 2) This newly developed TRCM model is much faster than the FEM for the same model so that it is cost-effective and practical for modeling long term (weeks to decades) thermal evolution in a BHE array; 3) Using the newly developed TRCM approach we studied the 'partition by partition' heating scenario and the results show a higher temperature concentrated in the center of the BHE array, a results desired for the system for reducing the loss of heat energy from the periphery of the BHE array.
\end{abstract}

Keywords: Seasonal thermal energy storage (STES), borehole heat exchanger (BHE), thermal resistance capacitance model (TRCM), heating scenario.

\section{Introduction}

Solar heating is a clear-energy technology that offers production of heat for residential space heating and domestic hot water production. However, the average power of solar energy has significant seasonal variation so that a cost effective long-term storage for regulating the supply-demand offset is highly desirable [1]. At the annual scale, the peak of solar energy harvest occurs during summer. Meanwhile, heat consumption is higher during winter season. Unfortunately, offsets and intermittence make achieving the potential of solar technologies relatively complex. Seasonal thermal energy storage (STES) with borehole heat exchanger (BHE) array is one of the most cost-effective way in energy storage of the industrial waste heat and solar heat collected in the summer season to supply residential heating in winter time [2].

Though the fundamental ground heat storage apparatus is still the borehole heat exchanger (BHE), a STES system is different from any other ground source heat pump or energy storage system in several major aspects. It elevates the ground soil temperature to a much higher level $\left(\sim 55^{\circ} \mathrm{C}\right)$ than traditional ground heat storage which only maintains it close to annual average temperature $\left(\sim 15^{\circ} \mathrm{C}\right)$. Hence, a relatively large storage volume $\left(\sim 0.5 \times 10^{6} \mathrm{~m}^{3}\right)$ is preferred to reduce the energy loss during the thermal storage periods ( $\sim 6$ months). Doing this will also greatly increase the thermal storage capability of the ground soil. The system will not use high-grade electricity to extract heat from the ground thermal storage. Instead, a smart design of the entire system and thermally driven absorption heat pump at the heating terminal station will be used. This approach allows a much lower return water temperature to be achieved $\left(\sim 20^{\circ} \mathrm{C}\right)$ so that direct heat exchange would allow obtaining heat through a variety of energy sources at different grades (ground heat exchangers, solar thermal, industrial waste heat, etc.) 
Modeling of the heat transport in the STES-BHE system has attracted significant attention from physicists and engineers. There are three fundamental approaches for heat transport modeling in this regard.

Analytical approach is the earliest technique to be used. Theoretical interpretations of the heat transfer phenomena within the ground surrounding the borehole are usually based on Lord Kelvin's infinite line-source theory is the basic technique for searching analytical solutions. Practical elaboration of this theory was given by Ingersoll and Zobel [3]. Using an analytical approach $\mathrm{Li}$ et al [4] developed a full-scale model for modeling the heat transfer in borehole exchangers from sub-hour to decades. The full scale model is a composite expression consisting of a medium line-source solution as the inner solution, a finite line source solution as the outer solution, and an infinite line-source solution. Next, they used the multi-stage model to combine the three separate solutions in a sequential way, i.e., the inner solution for the short-time scale, the conventional infinite line-source solution for the intermediate time scale, and the outer solution for the long-time scale.

On the opposite end of the spectrum of heat transport modeling is the widely used finite element method (FEM) (e.g., [5]). The thermal effect on the subsurface of a large borehole thermal energy store has been investigated by Mielke et al [6] with the use of the finite element model to validate the model with measured data from a 2-year operation period. The thermal changes in the subsurface have been predicted by simulation for a 30-year operation period. The model is based on three 80-m core sections drilled in Triassic carbonates, which have been analyzed in detail with respect to lithology, facies, and thermal and hydraulic parameters.

The thermal resistance capacitance model (TRCM) technique can provide reasonably accurate modeling results, but with much less stress in computer time and memory requirement. Assuming stationary conditions, Eskilson and Claesson [7] proposed to model the heat transport process in the borehole using a Delta-circuit analogy. This approach can model the long-term heat transfer phenomena and disregard the transient effects of heat and mass transport in the borehole and hence are not suitable for the prediction of the short-time behavior. In order to combine the advantages of both types of models, Bauer et al [8] developed a two-dimensional (2D) thermal resistance and capacity models to include the effects of the ground formation outside of the borehole, but limited with only a single BHE. Pasquier [9] developed further with TRCM to include the vertical evolution of temperature, also for a single BHE. A relatively more comprehensive review of all the modeling methods can be found from Ruan and Horton [10].

In this paper we extended the TRCM approach into a $2 \mathrm{D}$ fashion to model the heat transport in a horizontal plane of the BHE array at certain depth to include the effect of multiple BHEs. In the following sections we focus on the development of the TRCM technique and discuss its applicability with cautions of the limitations. We start with the hexagon pattern TRCM for a single borehole, then expand it to honey comb patterns to account for the contribution of multiple BHEs. The approach is tested by modeling the 'partition to partition' heating scenario. The results show that that approach can focus the high temperature to the center of the BHE array and increase the thermal energy storage efficiency by reducing the loss of heat from the periphery of the storage area.

\section{Development of the Thermal Resistance Capacitance Model (TRCM)}

Despite modern and powerful computers and the possibility of parallel computing fully discretized models lead to extensive computation times. Especially parameter estimation procedures with various iterations can be an unsolvable task applying fully discretized models. Additionally, the effort involved in doing the modeling work can be substantial. The TRCM model is one of the most effective way to describe the heat and transfer processes of BHE. One important analytical approach was shown by Eskilson and Claesson [7].

The criterion for the validity to utilize the lumped resistance-capacitance method is necessity of the Biot Number (Bi) to be much less than unity [11]. The extreme is that that lumped resistance-capacitance model is exact when $\mathrm{Bi}$ reaches zero, so that smaller $\mathrm{Bi}$ means better approximation to the real solution. The physical interpretation for this criterion is that we need the temperature change caused by the conduction in the solid is relatively small in comparison with the temperature change caused by the convection in the fluid. This is exactly the case for the borehole heat exchange system used for thermal energy storage with which the heat flow in the tubes is convective and have larger temperature change, while the dissipation of heat in the solid ground is conductive, and induces less temperature change.

\section{Basic TRCM Model of a U-Tube Borehole}

A basic TRCM model developed by Bauer et al [8], which represents a horizontal slice of the borehole configuration as shown in Fig. 1 as shown below, is the starting point for a novel TRCM model configuration developed in this paper. 


\section{Borehole and U-tube viewed from horizontal plane}

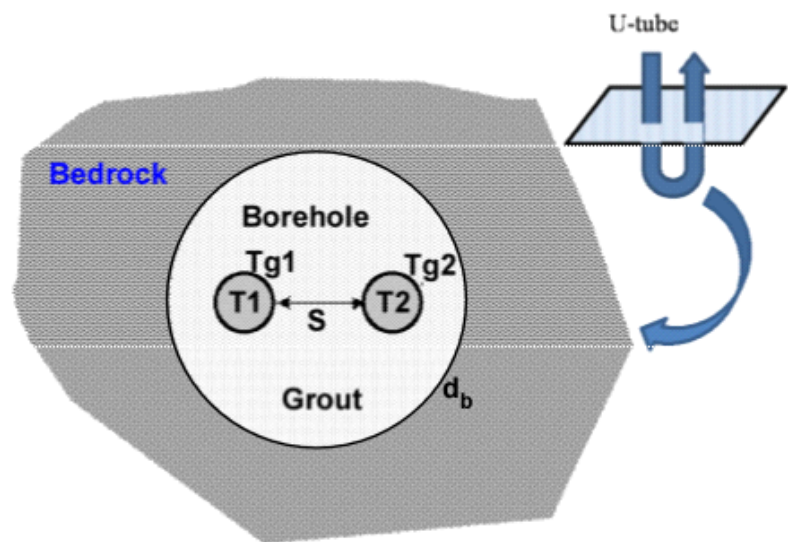

(a)

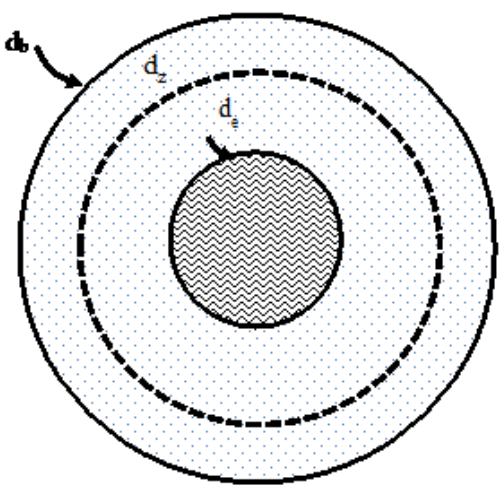

(b)

Fig. 1: Illustration of the BHE in a horizontal plane cross-section (a) and its equivalent thermal condition (b).

The equivalent diameters for a cross-section of a BHE as shown in Fig. $1 \mathrm{~b}$ are defined as follows:

$$
d_{e q}=\sqrt{2} \cdot d_{a}, \quad d_{z}=\frac{\sqrt{d_{b}^{2}+d_{e q}^{2}}}{2}
$$

where $d_{a}$ is the outer diameter of the U-tube, $d_{b}$ is the diameter of the borehole, $d_{e q}$ is the equivalent cross-section area of the U-tube, and $d_{z}$ is the equivalent diameter of the center of grout mass.

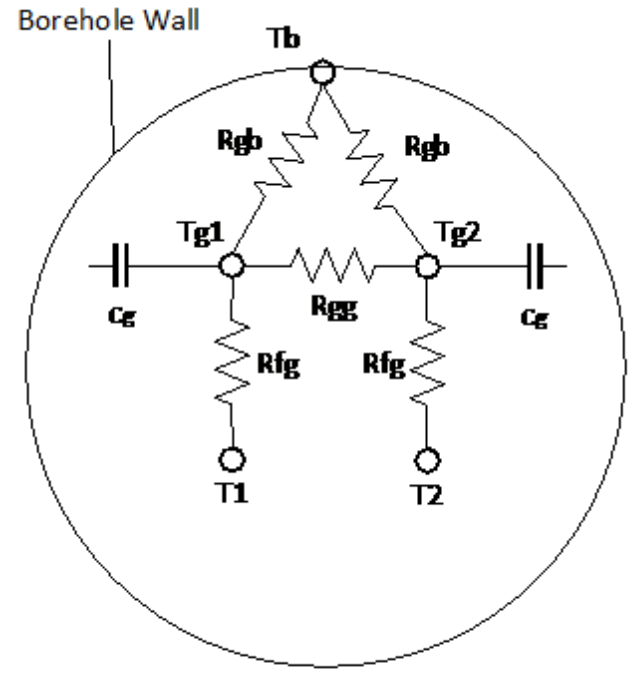

(a)

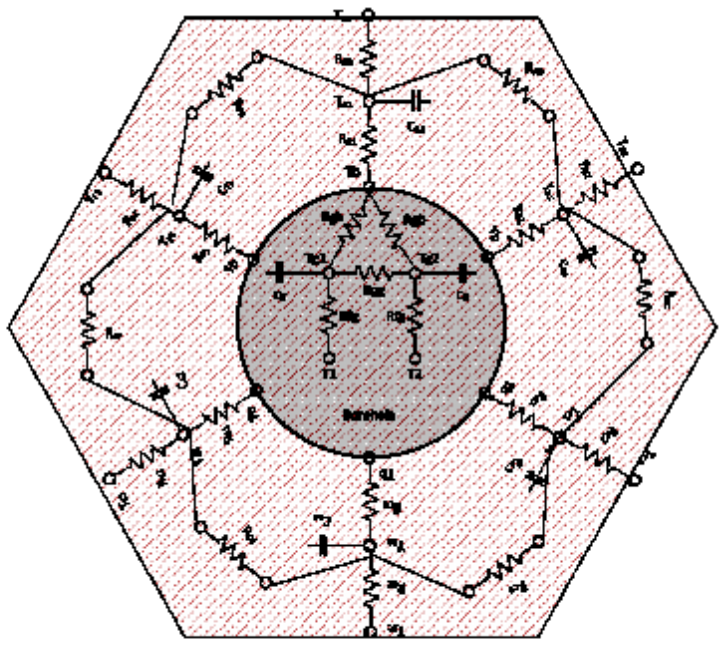

(b)

Fig. 2: The TRCM model for the inner borehole (a) and the hexagonal TRCM model with the inclusion of ground formation outside of the borehole with the potential to interconnect other hexagons to form a BHE array (b).

To validate the TRCM approach for modeling the heat transport for borehole heat exchangers, we compared the temperature field inside a borehole by using both the finite element method and the TRCM. The results are shown below as Fig. 3. 


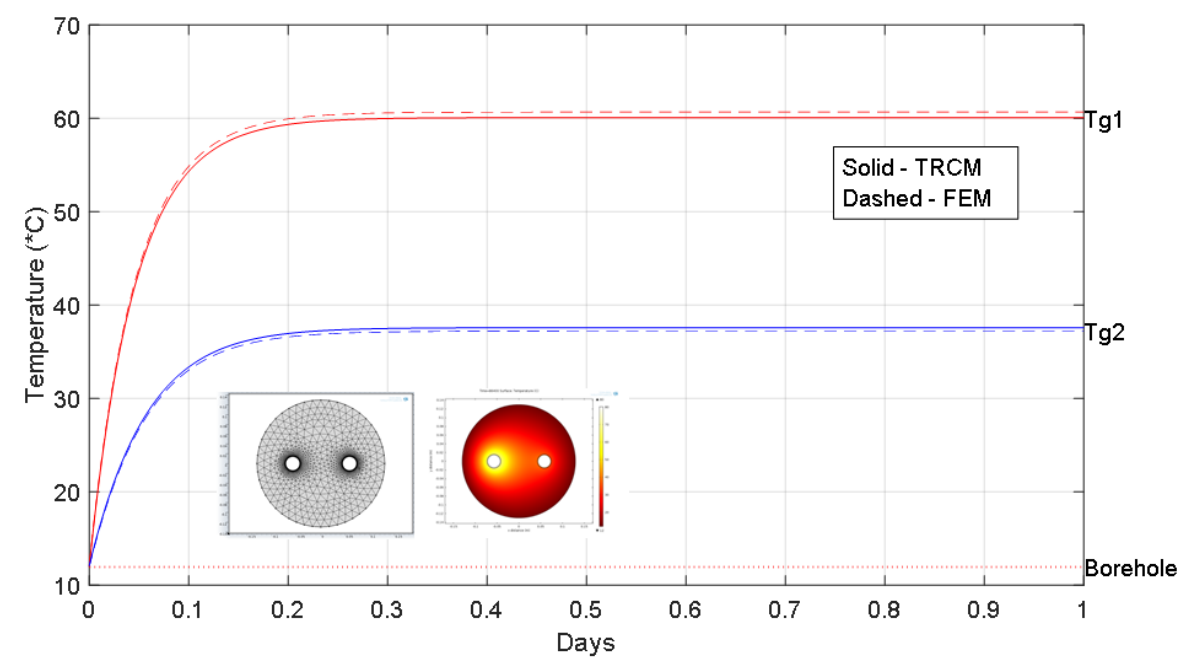

Fig. 3: Comparison of the temperature in the tube, the borehole by using the finite element and TRCM techniques.

The curve of $\mathrm{T}_{\mathrm{g} 1}$ and $\mathrm{T}_{\mathrm{g} 2}$ are the temperature of the outer diameter in the grout close to the inlet and outlet $\mathrm{U}$-tube, respectively. It is clear that the temporal variation of the temperatures from FEM and TRCM are closely in agreement with each other, with a difference of no more than $1 \%$. Considering the computation cost and the need to scale to a much larger model, apparently TRCM is a quite cost-effective approach for a heat transport simulation problem like those in the BHE array of a STEM system.

\section{TRCM Model of a U-Tube Borehole Array}

Based on the single hexagon element of the TRCM model for a single borehole, we can multiple it and connect all the hexagon elements together to form a honey comb type collections to model the heat transport in a BHE array, as shown in Fig. 4 below. Compared with the single hexagon model the size of the ODE equation is much larger. But with the computation capacity of the modern computers it is still very fast in comparison with using the finite element modeling technique.

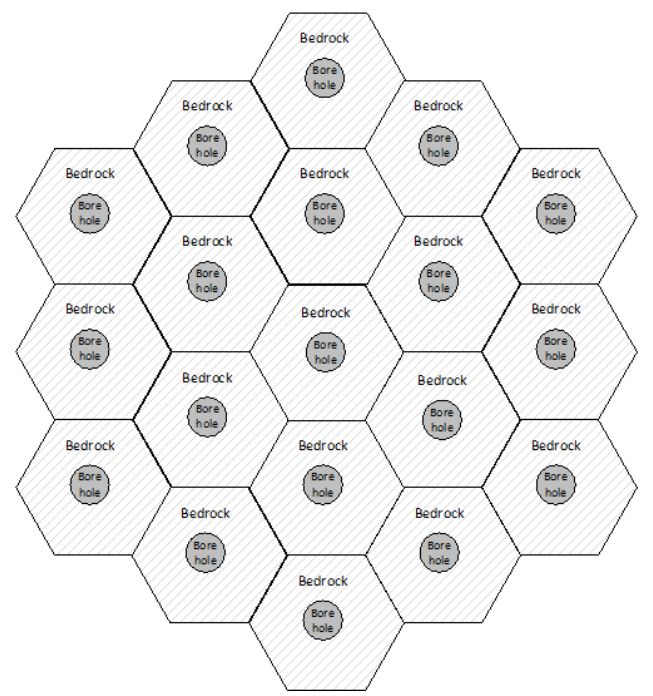

Fig. 4: The honey comb model of the BHE array consisted of single hexagon BHEs.

Using 19 hexagon elements we modelled the temperature evolution in a BHE array shown in Section 5 below. 


\section{Preliminary Modeling Results}

Based on the thermal parameter of the formation in a real STES site the average thermal conductivity in the project area is $0.852 \mathrm{~W} / \mathrm{m}^{\circ} \mathrm{C}$, and the average thermal capacity is $1.655 \times 10^{6} \mathrm{~J} / \mathrm{m}^{3 \circ} \mathrm{C}$. The average initial temperature is $12{ }^{\circ} \mathrm{C}$ we modelled the heating scenario of a 'partition by partition' fashion proposed by the designer with the objective to focus the high temperature in the center of the BHE array. Our simulation results are shown below in Fig. 5

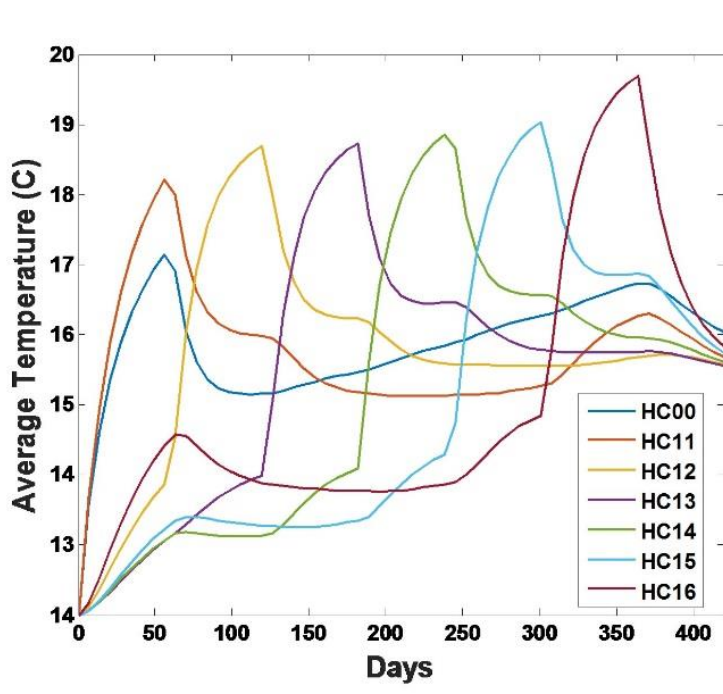

(a)

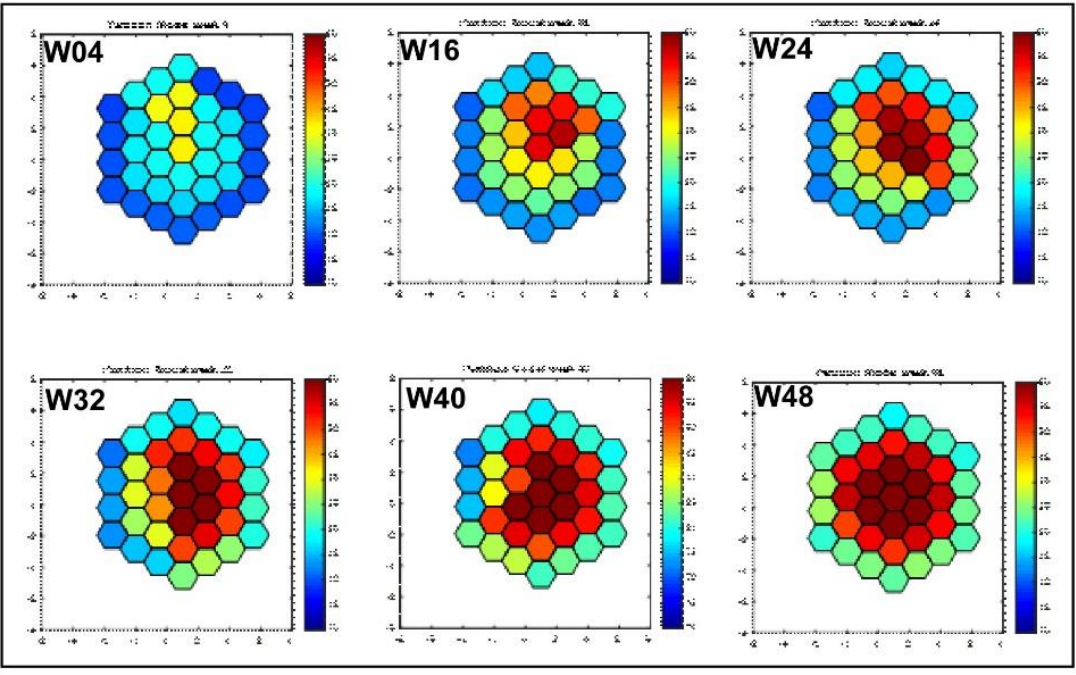

(b)

Fig. 5: TRCM modeling of the 'partition by partition' heating scenario with the heating history shown in (a) and the snapshots of temperature field show in (b).

In Fig. 5a is the heating history in the given U-tube inlet to show the turning on and turning off of the heat supply. In Fig. $5 \mathrm{~b}$ we show the snapshots of formation temperature field at Week 4, 16, 24, 32, 40, and 48. It is obvious that the high temperature has been focuses in the central part of the BHE array. By taking this heating scenario the storage efficiency is increased by reducing the loss of heat from the periphery of the BHE array boundary.

\section{Conclusion}

In this paper we presented a novel approach to extend the classic TRCM model technique in 2D to enable us to model the temperature field evolution in a horizontal plane of the ground formations within a BHE array for thermal energy storage. Upon comparison with the finite element method, which is the most widely used numerical simulation technique, the proposed approach is much faster, and request much less memory; so that it is cost-effective and practical for modeling long term (weeks to decades) thermal evolution in a BHE array. We have validated the approach by looking into a heating scenario that is proposed for a real-world thermal energy storage project that uses a 'partition by partition' strategy. The modeling results indicated that the proposed heating scenario does focus the high temperature into the central area of the BHE array, an ideal temperature distribution pattern to reduce the heat loss and increase the cost effectiveness of the STES system. We plan to use the developed TRCM approach to study more heating scenarios and include the effects of the transient changes of formation thermal environment in the near future.

\section{Acknowledgements}

We are grateful to Professor Xudong Yang and Dr. Fang Guo of the Department of Building Technology and Science, School of Architecture, Tsinghua University provided the design and plans of a pilot project of "Study of the integration of industrial waste heat and solar energy for long-term thermal storage that stimulated the study presented in this paper. 


\section{References}

[1] Energy Solutions: REHAU Group. Geothermal Probes - Seasonal Heat Storage [Online]. Available: http://www.rehau-energy-solutions.com/EN/saisonalspeicher.html

[2] Energy Solutions: REHAU Group. REHAU Presentation Underground thermal Energy Storage [Online]. Available: http://www.rehau-energy-solutions.com/EN/saisonalspeicher.html

[3] L. R. Ingersoll and A. C. Zobel, Heat conduction with engineering and geological application - $2^{\text {nd }}$ ed. New York: McGrawHill, 1954.

[4] M. Li, P. Li, V. Chan and A. Lai, "Full-scale temperature response function (G-function) for heat transfer by borehole ground heat exchangers (GHEs) from sub-hour to decades," Applied Energy, vol. 136, pp. 197-205, 2014.

[5] D. O. Schulte, W. Rühaak, S. Chauhan, B. Welsch and I. Sass, "A MATLAB toolbox for optimization of deep borehole heat exchanger arrays," in Proceedings of the World Geothermal Congress 2015, Melbourne, Australia, 2015.

[6] P. Mielke, D. Bauer, S. Homuth, A. E Götz and I. Sass, "Thermal effect of a borehole thermal energy store on the subsurface," Geothermal Energy, vol. 2, no. 5, 2014.

[7] P. Eskilson and J. Claesson, "Simulation model for thermally interacting heat extraction boreholes," Numerical Heat Transfer, vol. 13, pp. 149-165, 1988.

[8] D. Bauer, W. Heideman, H. Muller-Stien and H. Dirersch, "Thermal resistance and capacity models for borehole heat exchangers," Int. J. Energy Res., vol. 35, pp. 312-320, 2011.

[9] P. Pasquier, "Stochastic interpretation of thermal response test with TRT-SInterp," Computers \& Geosciences, vol. 75, pp. 73-87, 2015.

[10] W. Ruan and W. T. Horton, "Literature Review on the Calculation of Vertical Ground Heat Exchangers for Geothermal Heat Pump Systems," in Proceedings of the International High Performance Buildings Conference, Paper 45, 2010.

[11] T. Sliwa and M. A. Rosen, "Natural and artificial methods for regeneration of heat resources for borehole heat exchangers to enhance the sustainability of underground thermal storages, A Review," Sustainability, vol. 7, pp. 13104-13125, 2015. 